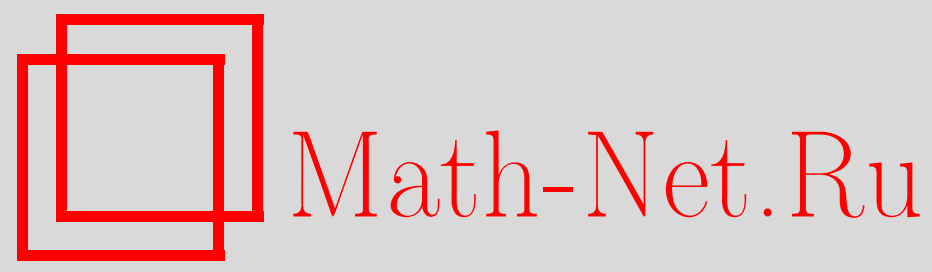

М. Г. Смирнова, Представление функции Грина уравнения теплопроводности на компактной группе Ли, Матем. заметки, 2000, том 67, выпуск 3, 397-413

DOI: https://doi.org/10.4213/mzm853

Использование Общероссийского математического портала Math-Net.Ru подразумевает, что вы прочитали и согласны с пользовательским соглашением http://www.mathnet.ru/rus/agreement

Параметры загрузки:

IP : 52.6 .47 .48

26 апреля 2023 г., 18:12:55

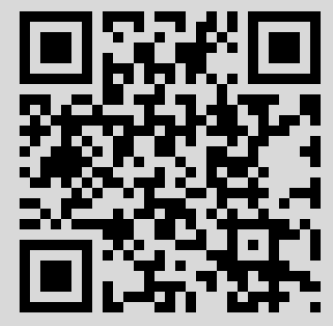




\title{
ПРЕДСТАВЛЕНИЕ ФУНКЦИИ ГРИНА УРАВНЕНИЯ ТЕПЛОПРОВОДНОСТИ НА КОМПАКТНОЙ ГРУППЕ ЛИ
}

\author{
М.Г. Смирнова
}

Получена явная формула, представляющая решение уравнения теплопроводности на компактной группе Ли в виде предела конечнократных сверток функции Грина для уравнения теплопроводности в евклидовом пространстве.

Библиография: 14 названий.

Основной результат работы можно рассматривать как методпостроения броуновского движения на произвольном компактном римановом многообразии, обладающим постоянной кривизной Риччи; он совершенно отличен от используемых в настояшее время подходов, ставших уже почти стандартными и основанных на применении теории полугрупп (см. [1], [2]) и стохастических дифференциальных уравнений (см., например, [3]-[7] и имеющиеся там ссылки).

Существует еще и совсем иной более алгебраический подход, применимый только к компактным группам Ли и дающий “явное" представление решений уравнения теплопроводности в виде бесконечного ряда по (выраженным весьма громоздкими формулами) собственным функциям оператора Бельтрами-Лапласа на группе, которые могут быть найдены методами теории представлений [8]. Значительное внимание, которое сейчас уделяется в литературе случайным процессам и дифференциальным уравнениям на компактных римановых многообразиях, объясняется, в частности, той ролью, которую они играют в квантовой теории (например, в теории струн и в теории калибровочных полей).

Представление решений эволюционных уравнений с частными производньми (относительно функций, определенных на евклидовых пространствах) в виде предела конечнократных интегралов было впервые получено Р. Фейнманом в его известной работе [9], в которой фактически была угадана формула Троттера. Точньй математический смысл рассуждениям Р. Фейнмана был придан Э. Нельсоном в [10]. Доказанную в работе формулу (без доказательства она была приведена в [11]) можно интерпретировать как распространение формулы Фейнмана на уравнение теплопроводности с сингулярньм потенциалом типа "регуляризованной” $\delta$-функции, сосредоточенным на многообразии, вложенном в подходящее евклидово пространство. Точная формулировка полученного результата следующая.

Пусть $G$ - компактная связная группа Ли, реализованная согласно теореме Петера-Вейля как подгруппа некоторой унитарной групшы $U(N)$ и, соответственно, как поверхность в пространстве $M \cong \mathbb{C}^{N^{2}}$ всех комплексных $N \times N$ матриц. Эрмитово ска- 
лярное произведение в $\mathbb{C}^{N^{2}}$, задаваемое формулой $(A, B)=\operatorname{tr} A B^{*}=\sum_{i, j} A_{i j} \bar{B}_{i j}$, инвариантно относительно действия грушшы $U(N)$.

Тогда решение уравнения теплопроводности

$$
\frac{\partial f}{\partial t}=\frac{1}{2} \Delta f,\left.\quad f\right|_{t=0}=f_{0},
$$

где $f: G \times[0, T] \rightarrow \mathbb{R}^{1}, f_{0} \in C^{2}(G)$, может быть записано в виде:

$$
\begin{aligned}
f(t, X)= & \lim _{\substack{\max h_{i} \rightarrow 0 \\
h_{1}+\cdots+h_{n}=t}} \int_{G} \cdots \int_{G} f_{0}\left(X_{1}\right) p\left(X_{1}, X_{2}, h_{1}\right) \\
& \times p\left(X_{2}, X_{3}, h_{2}\right) \cdots p\left(X_{n}, X, h_{n}\right) d X_{1} \cdots d X_{n}
\end{aligned}
$$

где

$$
p(X, Y, h)=\frac{C_{h}}{(\sqrt{2 \pi h})^{m}} e^{-\|X-Y\|^{2} /(2 h)}, \quad \int_{G} \frac{C_{h}}{(\sqrt{2 \pi h})^{m}} e^{-\|X-E\|^{2} /(2 h)} d X=1 .
$$

Здесь $\Delta=-\Delta_{B L}, \Delta_{B L}-$ оператор Бельтрами-Лапласа (см., например, [12]); $\|X\|=$ $\sqrt{(X, X)}-$ норма в пространстве $M, m=\operatorname{dim} G-$ размерность $G, E$ - единица группы $G$ (единичная матрица).

1. Предварительные сведения. Пусть $\nu_{G}$ и $\nu_{T_{E}} G-$ меры на групше $G$ и на касательном пространстве $T_{E} G$ соответственно, индуцированные вложением $G$ и $T_{E} G$ в евклидово пространство $M$ с мерой Лебега. Из инвариантности скалярного произведения следует инвариантность меры $\nu_{G}$ относительно умножений в группе. Везде в дальнейшем при интегрировании по группе $G$ и пространству $T_{E} G$ подразумевается интегрирование по мере $\nu_{G}$ и $\nu_{T_{E}} G$ соответственно.

Для доказательства основной формулы нам потребуется ряд вспомогательных утверждений.

ПРЕДЛОЖЕНИЕ 1 (см. [13, 11]). Пусть $G$ - компактная матричная группа Ли, наделенная римановой метрикой, инвариантной относительно групповой операиии. Тогда геодезические этой метрики имеют вид $B e^{A t}$, где $B \in G, A \in T_{E} G-$ касательному пространству в единице $\kappa$ группе $G$, причем если $\|A\|=1$, то $t$ натуральный параметр.

ПРЕДЛОЖЕНИЕ 2. Пусть $F^{n}-$ риманово многообразие, $и \varphi \in C^{2}\left(F^{n}\right)$. Тогда

$$
\Delta \varphi(x)=\left.\sum_{l=1}^{n} \frac{d^{2} \varphi\left(x_{l}(t)\right)}{d t^{2}}\right|_{t=0}
$$

где $x_{l}(t)$ - семейство геодезических, выходящих из точки $x$ и таких, что векторы $\left.\dot{x}_{l}(t)\right|_{t=0}$ образуют ортонормированный базис в касательном пространстве $\kappa F_{n}$ в точке $x$.

ПРЕДЛОЖЕНИЕ 3. Пусть $G$ - компактная группа Ли. Существует ограниченная симметричная окрестность нуля $U$ в $T_{E} G$ такая, что әкспоненциальное отображсение $\exp : Y \mapsto e^{Y}\left(T_{E} G \rightarrow G\right)$ взаимно однозначно отображсает $U$ на $\exp (U)$ $u \nu_{G}(G \backslash \exp (U))=0$. 
ДокАЗАТЕЛЬСтво. Можно считать $G$ подгруппой группы унитарных матрищ $U(n)$ для некоторого $n$. Тогда $T_{E} G$ - подпространство в пространстве $\mathscr{K}_{n}$ всех косоэрмитовых матриц порядка $n$. Введем в пространстве $\mathscr{K}_{n}$ норму

$$
\|A\|_{1}=\max _{x \in \mathbb{C}^{n}} \frac{\|A x\|}{\|x\|} .
$$

Множество $U=\left\{X \in T_{E} G:\|X\|_{1}<\pi\right\}$ ограничено, открыто и симметрично; экспоненциальное отображение сюръективно отображает его замыкание $\bar{U}=\left\{X \in T_{E} G\right.$ : $\left.\|X\|_{1} \leqslant \pi\right\}$ на группу $G$. Инъективность экспоненциального отображения на $U$ проверяется элементарно. Поскольку якобиан экспоненциального отображения ограничен на $\bar{U}$ и множество $\bar{U} \backslash U$ имеет лебегову меру нуль, то $\nu_{G}(G \backslash \exp (U))=0$. Предложение доказано.

Везде в дальнейшем мы будем считать, что окрестность $U \subset T_{E} G$, удовлетворяюшая условиям предложения 3 , фиксирована. Отметим, что для любой функции $\varphi \in C(G)$

$$
\int_{G} \varphi(X) d X=\int_{U} \psi(Y)|\mathscr{J}(Y)| d Y,
$$

где $\psi(Y)=\varphi\left(e^{Y}\right), \mathscr{J}$ - якобиан отображения $\exp : T_{E} G \rightarrow G$.

Отметим также, что $\mathscr{J}(-X)=\mathscr{J}(X)$, и, поскольку $\mathscr{J}(0)=1$, в окрестности нуля в $T_{E} G$ вьполняется неравенство $1-c\|X\|^{2} \leqslant|\mathscr{J}(X)| \leqslant 1+c\|X\|^{2}$.

Положим

$$
p_{h}(X)= \begin{cases}p\left(e^{X}, E, h\right)|\mathscr{J}(X)|, & X \in U ; \\ 0, & X \in T_{E} G \backslash U .\end{cases}
$$

Тогда

$$
\int_{T_{E} G} \varphi\left(e^{Y}\right) p_{h}(Y) d Y=\int_{G} \varphi(X) p(X, E, h) d X,
$$

где $\varphi \in C(G)$. Символом $\xi_{h}$ будем обозначать случайную величину, принимающую значения в $T_{E} G$, распределение которой имеет плотность $p_{h}$ относительно меры $\nu_{T_{E}} G$. Случайная величина $e^{\xi_{h}}$ принимает значения в $G$, и ее распределение согласно (1.1) имеет плотность $p(X, E, h)$ относительно меры $\nu_{G}$. Заметим, что $p(A X, A Y, h)=p(X, Y, h)$ и

$$
\int_{G} \varphi(X) d X=\int_{G} \varphi(X Y) d X
$$

поскольку форма объема $d X$, задаваемая метрикой, индуцированной вложением $G \subset M$, инвариантна относительно групповой операции. Эти два факта будут в дальнейшем использоваться без пояснений. Символом $\mathrm{E} \xi$ обозначается математическое ожидание случайной величины $\xi$.

Лемма 1. Пусть $\xi_{1}$ и $\xi_{2}-$ две независимье случайные величины, принимающие значения в $G$, с плотностями $p_{\xi_{1}}$ и $p_{\xi_{2}}$ относительно мерь $\nu_{G}$. Тогда случайная величина $\xi_{1} \xi_{2}$ имеет плотность

$$
p_{\xi_{1} \xi_{2}}(X)=\int_{G} p_{\xi_{1}}\left(X Y^{-1}\right) p_{\xi_{2}}(Y) d Y .
$$


СлЕДСТВИЕ 1. Случайная величина $e^{\xi_{h_{1}}} \cdots e^{\xi_{h_{n}}}$ имеет плотность

$$
p\left(X_{1}\right)=\int_{G} \cdots \int_{G} p\left(X_{1}, X_{2}, h_{1}\right) p\left(X_{2}, X_{3}, h_{2}\right) \cdots p\left(X_{n}, E, h_{n}\right) d X_{2} \cdots d X_{n} .
$$

Кроме того

$$
\begin{aligned}
\mathrm{E} \varphi\left(e^{\xi_{h_{1}}} \cdots e^{\left.\xi_{h_{n}} X\right)=}\right. & \int_{G} \cdots \int_{G} \varphi\left(X_{1}\right) p\left(X_{1}, X_{2}, h_{1}\right) \\
& \times p\left(X_{2}, X_{3}, h_{2}\right) \cdots p\left(X_{n}, X, h_{n}\right) d X_{1} d X_{2} \cdots d X_{n} .
\end{aligned}
$$

2. Случайные величины, принимающие значения в группе $G$. Непосредственно проверяется, что для нормы в пространстве $M \cong C^{N^{2}}$, индуцированной скалярным произведением

$$
(A, B)=\sum_{i, j=1}^{N} A_{i j} \bar{B}_{i j}
$$

выполняется неравенство $\|A B\| \leqslant\|A\|\|B\|$. В дальнейшем этот факт будет использоваться без пояснений.

Утверждения следующих четырех технических лемм проверяются непосредственно.

Лемма 2. Пусть $X \in T_{E} G$. Тогда $\|X\|^{2}-\left\|e^{X}-E\right\|^{2} \leqslant O\left(\|X\|^{4}\right)(\|X\| \rightarrow 0)$.

ЛЕмма 3. Справедлива оиенка $\left|C_{h}-1\right|=O(h)(h \rightarrow 0)$.

ЛЕМма 4. Выполняются соотношения

$$
\begin{gathered}
\frac{1}{\sqrt{2 \pi h}} \int_{\mathbb{R}^{1}} x^{2 k} e^{-|x|^{2} /(2 h)} d x=\frac{(2 k) !}{2^{k} k !} h^{k} \\
\frac{1}{(\sqrt{2 \pi h})^{m}} \int_{\mathbb{R}^{m}}\left(x_{1}^{2}\right)^{\alpha_{1}} \cdots\left(x_{m}^{2}\right)^{\alpha_{m}} e^{-\|x\|^{2} /(2 h)} d x=\frac{\left(2 \alpha_{1}\right) ! \cdots\left(2 \alpha_{m}\right) !}{2^{\alpha_{1}+\cdots+\alpha_{m} \alpha_{1} ! \cdots \alpha_{m} !} h^{\alpha_{1}+\cdots+\alpha_{m}}} \\
\frac{1}{(\sqrt{2 \pi h})^{m}} \int_{\mathbb{R}^{m}}\|x\|^{2 k} e^{-\|x\|^{2} /(2 h)} d x=\frac{h^{k} k !}{2^{k}} \sum_{\alpha_{1}+\cdots+\alpha_{m}=k} \frac{\left(2 \alpha_{1}\right) ! \cdots\left(2 \alpha_{m}\right) !}{\left(\alpha_{1} ! \cdots \alpha_{m} !\right)^{2}} \leqslant c^{k} h^{k} k !, \\
\frac{1}{(\sqrt{2 \pi h})^{m}} \int_{\mathbb{R}^{m}}\|x\|^{2 k-1} e^{-\|x\|^{2} /(2 h)} d x \leqslant c^{k-1 / 2} h^{k-1 / 2} k !,
\end{gathered}
$$

әде с зависит только от $\mathrm{m}$.

ЛЕмма 5. Справедливы оценки

$$
\begin{gathered}
\int_{U}\|X\|^{k} p_{h}(X) d X \leqslant c(m)^{k} \frac{1}{(\sqrt{2 \pi h})^{m}} \int_{T_{E} G}\|X\|^{k} e^{-\|X\|^{2} /(2 h)} d X \\
\int_{U}\left(X_{1}^{2}\right)^{\alpha_{1}} \cdots\left(X_{m}^{2}\right)^{\alpha_{m}} p_{h}(X) d X \leqslant \frac{c(m)^{\alpha}}{(\sqrt{2 \pi h})^{m}} \int_{T_{E} G}\left(X_{1}^{2}\right)^{\alpha_{1}} \cdots\left(X_{m}^{2}\right)^{\alpha_{m}} e^{-\|X\|^{2} /(2 h)} d X
\end{gathered}
$$

где $m=\operatorname{dim} G, \alpha=2\left(\alpha_{1}+\cdots+\alpha_{m}\right), X_{1}, \ldots, X_{m}-$ координаты вектора $X \boldsymbol{в}$ некотором ортонормированном базисе в $T_{E} G$.

Из лемм $2-5$ и оценки $1-c\|X\|^{2} \leqslant|\mathscr{J}(X)| \leqslant 1+c\|X\|^{2}$ следует 
ЛЕмма 6. Выполняется асимптотическая формула

$\left|\int_{U}\|X\|^{k} p_{h}(X) d X-\frac{1}{(\sqrt{2 \pi h})^{m}} \int_{\mathbb{R}^{m}}\|X\|^{k} e^{-\|X\|^{2} /(2 h)} d X\right|=O\left(h^{k / 2+1}\right) \quad(h \rightarrow 0)$, где константа в $O\left(h^{k / 2+1}\right)$ не зависит от $h u k$.

TЕоремА 1. Пусть $\xi_{h_{1}^{i}}, \ldots, \xi_{h_{n_{i}}^{i}}-$ независимые случайные величины с плотнос$\operatorname{ms\mathcal {M}u} p_{h_{1}^{i}}, \ldots, p_{h_{n_{i}}^{i}} u$

$$
\max _{1 \leqslant j \leqslant n_{i}} h_{j}^{i} \rightarrow 0 \quad(i \rightarrow \infty), \quad \sum_{j=1}^{n_{i}} h_{j}^{i}=s .
$$

Тогда $\xi_{h_{1}^{i}}+\cdots+\xi_{h_{i}^{i}} \stackrel{w}{\rightarrow} \zeta_{s}(i \rightarrow \infty)$, әде $\zeta_{s}-$ гауссовская случайная величина в $T_{E} G$ c матрицей ковариачий $s E_{m}$, где $E_{m}$ - единичная матрица размера $m \times m$.

ДокАЗАТЕльСТво. Пусть $\varphi_{\xi_{h}}$ - характеристическая функция случайной величины $\xi_{h}$. Тогда

$$
\varphi_{\xi_{h}}(t)=\int_{T_{E} G} e^{i(t, X)} p_{h}(X) d X=\sum_{k=0}^{\infty} \frac{i^{k}}{k !} \int_{T_{E} G}(t, X)^{k} p_{h}(X) d X
$$

$p_{h}(-X)=p_{h}(X)$, так как $p\left(e^{-X}, E, h\right)=p\left(e^{-X} e^{X}, e^{X}, h\right)=p\left(E, e^{X}, h\right), \mathscr{J}(-X)=$ $\mathscr{J}(X)$. Следовательно,

$$
\int_{T_{E} G}(t, X) p_{h}(X) d X=0 .
$$

По лемме 6

$$
\begin{aligned}
& \left|\frac{1}{2} \int_{T_{E} G}(t, X)^{2} p_{h}(X) d X-\frac{h}{2} \sum_{i=1}^{m} t_{i}^{2}\right| \\
& \quad \leqslant\left|\frac{1}{2} \int_{T_{E} G}\|t\|^{2}\|X\|^{2}\left(p_{h}(X)-\frac{1}{(\sqrt{2 \pi h})^{m}} e^{-\|X\|^{2} /(2 h)}\right) d X\right|=\|t\|^{2} O\left(h^{2}\right) \quad\left(\begin{array}{r}
(h \rightarrow 0) . \\
(2.2)
\end{array}\right.
\end{aligned}
$$

Пользуясь леммами 4 и 5 , получаем

$$
\begin{aligned}
& \left|\sum_{k=3}^{\infty} \frac{i^{k}}{k !} \int_{T_{E} G}(t, X)^{k} p_{h}(X) d X\right| \leqslant\left|\sum_{k=3}^{\infty} \frac{\|t\|^{k}}{k !} \int_{T_{E} G}\|X\|^{k} p_{h}(X) d X\right| \\
& \leqslant \sum_{k=2}^{\infty} \frac{\|t\|^{2 k}}{(2 k) !} c^{2 k} h^{k} k !+\sum_{k=1}^{\infty} \frac{\|t\|^{2 k+1}}{(2 k+1) !} c^{2 k+1} h^{k+1 / 2}(k+1) ! \\
& \leqslant O\left(\|t\|^{3} h^{3 / 2}\right) \quad\left(\|t\| h^{1 / 2} \rightarrow 0\right) .
\end{aligned}
$$

Учитывая $(2.1),(2.2)$ и (2.3), имеем

$$
\varphi_{\xi_{h}}(t)=1-\frac{h\|t\|^{2}}{2}+O\left(h^{3 / 2}\|t\|^{3}\right)+O\left(\|t\|^{2} h^{2}\right) .
$$


Пусть теперь

$$
\xi^{i}=\sum_{j=1}^{n_{i}} \xi_{h_{j}^{i}}
$$

Тогда

$$
\begin{aligned}
\ln \varphi_{\xi^{i}} & =\sum_{j=1}^{n_{i}} \ln \varphi_{\xi_{h_{j}^{i}}}=-\frac{1}{2} \sum_{j=1}^{n_{i}} h_{j}^{i}\|t\|^{2}+O\left(\sum_{j=1}^{n_{i}}\left(h_{j}^{i}\right)^{3 / 2}\|t\|^{3}\right)+O\left(\sum_{j=1}^{n_{i}}\left(h_{j}^{i}\right)^{2}\|t\|^{2}\right) \\
& \rightarrow-\frac{1}{2} h\|t\|^{2} \quad(i \rightarrow \infty) .
\end{aligned}
$$

Из известной теоремы о сходимости распределений следует, что $\xi^{i} \stackrel{w}{\rightarrow} \eta(i \rightarrow \infty)$.

СЛЕДСТВИЕ 2. Пусть $\varphi \in C(G), \mu_{1}, \mu_{2}$ - случайные величины, принимающие значения в группе $G$. Пусть $h_{1}+\cdots+h_{n}=h-$ разбиение отрезка $[0, h]$. Если $\xi_{h_{1}}, \ldots, \xi_{h_{n}}$ с плотностями $p_{h_{1}}, \ldots, p_{h_{n}}$ таковы, что $\mu_{1}, \mu_{2}, \xi_{h_{1}}, \ldots, \xi_{h_{n}}-$ независимые случайные величины, $и \zeta_{h}$ - гауссовская случайная величина в $T_{E} G$ с матричей ковариаций $h E_{m}$ такая, что случайные величины $\mu_{1}, \mu_{2}, \zeta_{h}$ независимы, то

$$
\left|\mathrm{E} \varphi\left(\mu_{1} e^{\xi_{h_{1}}+\cdots+\xi_{h_{n}}} \mu_{2}\right)-\mathrm{E} \varphi\left(\mu_{1} e^{\zeta_{h}} \mu_{2}\right)\right| \rightarrow 0 \quad\left(\max _{1 \leqslant i \leqslant n}\left|h_{i}\right| \rightarrow 0\right)
$$

равномерно по $h_{i} u \mu_{1}, \mu_{2}$.

ДокАЗАТЕЛЬСТво. Из теоремы 1 вытекает, что для любых $X, Y \in G$

$$
\left|\mathrm{E} \varphi\left(X e^{\xi_{h_{1}}+\cdots+\xi_{h_{n}}} Y\right)-\mathrm{E} \varphi\left(X e^{\zeta_{h}} Y\right)\right| \rightarrow 0 \quad\left(\max _{1 \leqslant i \leqslant n}\left|h_{i}\right| \rightarrow 0\right)
$$

Поскольку семейство функций $\left\{f_{\mu}(X, Y)=\mathrm{E} \varphi(X \mu Y): \mu\right.$ - случайная величина со значениями в $G$ \} равностепенно равномерно непрерывно на компакте $G \times G$, сходимость в (2.4) равномерна по $X, Y \in G$, что и влечет нужное нам утверждение.

Лемма 7. Пусть $\xi_{h_{1}}, \ldots, \xi_{h_{n}}$ независимьи, $h_{i}>0 u h_{1}+\cdots+h_{n}=h$. Тогда

$$
\mathrm{E}\left\|\xi_{h_{1}}+\cdots+\xi_{h_{n}}\right\|^{2 k} \leqslant \frac{(2 k) !}{k !} c^{k} h^{k}, \quad \mathrm{E}\left\|\xi_{h_{1}}+\cdots+\xi_{h_{n}}\right\|^{2 k-1} \leqslant \frac{(2 k) !}{k !} c^{k-1 / 2} h^{k-1 / 2}
$$

где константа с зависит только от выбора группь $G$.

ДокАЗАТЕЛЬСТво. Пусть $\xi=\xi_{h_{1}}+\cdots+\xi_{h_{n}}$. Фиксируем произвольный ортонормированньй базис в $T_{E} G$. В этом базисе $\xi=\left(\xi^{1}, \ldots, \xi^{m}\right), \xi_{h_{j}}=\left(\xi_{h_{j}}^{1}, \ldots, \xi_{h_{j}}^{m}\right)$, $\xi^{i}=\xi_{h_{1}}^{i}+\cdots+\xi_{h_{n}}^{i}$. Из лемм 4 и 5 следует, что

$$
\mathrm{E}\left(\xi^{i}\right)^{2 k} \leqslant \frac{(2 k) !}{k !} c^{k} h^{k}
$$

где $c$ зависит только от групшы $G$. По неравенству Гёльдера из (2.5) вытекает, что

$$
\mathrm{E}\left(\xi^{1}\right)^{2 k_{1}} \cdots\left(\xi^{m}\right)^{2 k_{m}} \leqslant \frac{(2 k) !}{k !} c^{k} h^{k}
$$


Следовательно,

$$
\mathrm{E}\|\xi\|^{2 k} \leqslant \frac{(2 k) !}{k !} m^{k} c^{k} h^{k}
$$

Для оценки $\mathrm{E}\|\xi\|^{2 k-1}$ снова воспользуемся неравенством Гёльдера и уже имеющейся оценкой для $\mathrm{E}\|\xi\|^{2 k}$ :

$$
\begin{aligned}
\mathrm{E}\|\xi\|^{2 k-1} & \leqslant\left(\mathrm{E}\|\xi\|^{2 k}\right)^{(2 k-1) /(2 k)} \leqslant\left(\frac{(2 k) !}{k !} m^{k} c^{k} h^{k}\right)^{(2 k-1) /(2 k)} \\
& \leqslant \frac{(2 k) !}{k !} m^{k-1 / 2} c^{k-1 / 2} h^{k-1 / 2}
\end{aligned}
$$

Лемма доказана.

Непосредственным следствием предыдущей леммы является

Лемма 8. Пусть $\xi_{h_{1}}, \ldots, \xi_{h_{n}}$ независимы $u h_{1}+\cdots+h_{n}=h$. Тогда

$$
\begin{gathered}
\mathrm{E}\left(\sum_{j=k}^{\infty} \frac{\left\|\xi_{h_{1}}+\cdots+\xi_{h_{n}}\right\|^{j}}{j !}\right)=O\left(h^{k / 2}\right), \\
\mathrm{E}\left(\frac{\left\|\xi_{h_{1}}+\cdots+\xi_{h_{n}}\right\|^{2}}{2 !}+\frac{\left\|\xi_{h_{1}}+\cdots+\xi_{h_{n}}\right\|^{3}}{3 !}+\cdots\right)^{2}=O\left(h^{2}\right), \\
\mathrm{E}\left(\left\|\xi_{h_{1}}+\cdots+\xi_{h_{n}}\right\|+\frac{\left\|\xi_{h_{1}}+\cdots+\xi_{h_{n}}\right\|^{2}}{2 !}+\cdots\right)^{2}=O(h) \quad(h \rightarrow 0),
\end{gathered}
$$

где константы в $O\left(h^{k / 2}\right), O\left(h^{2}\right), O(h)$ зависят только от группь $G$.

ЛЕмма 9. Пусть $\varphi \in C^{1}(M), \xi_{h_{1}}, \xi_{h_{2}}$ - независимые случайные величины $c$ плотностями $p_{h_{1}}, p_{h_{2}}$. Пусть $\xi_{h}$ - случайная величина с плотностью $p_{h}, h=$ $h_{1}+h_{2}$. Тогда $\left|\mathrm{E} \varphi\left(e^{\xi_{h_{1}}+\xi_{h_{2}}}\right)-\mathrm{E} \varphi\left(e^{\xi_{h}}\right)\right| \leqslant l(\varphi) O\left(h^{1 / 2}\right)$, гдe

$$
l(\varphi)=\max _{X \in B}\left\|\varphi^{\prime}(X)\right\|, \quad\left\|\varphi^{\prime}(X)\right\|=\sup _{Y \in M} \frac{\left|\varphi^{\prime}(X) Y\right|}{\|Y\|}
$$

В - шар в $M$ с иентром в нуле, содержащий компактную группу Ли $G$, и константа в $O\left(h^{1 / 2}\right)$ зависит только от группь $G$.

ДокАЗАТЕЛЬСТво. Очевидно,

$$
\left|\mathrm{E} \varphi\left(e^{\xi_{h_{1}}+\xi_{h_{2}}}\right)-\mathrm{E} \varphi\left(e^{\xi_{h}}\right)\right| \leqslant\left|\mathrm{E} \varphi\left(e^{\xi_{h_{1}}+\xi_{h_{2}}}\right)-\varphi(E)\right|+\left|\varphi(E)-\mathrm{E} \varphi\left(e^{\xi_{h}}\right)\right| .
$$

Поскольку

$$
\varphi\left(e^{\xi_{h_{1}}+\xi_{h_{2}}}\right)-\varphi(E)=\varphi^{\prime}(\zeta)\left(e^{\xi_{h_{1}}+\xi_{h_{2}}}-E\right)
$$

где $\zeta$ - точка из отрезка $\left[e^{\xi_{h_{1}}+\xi_{h_{2}}}, E\right]$, согласно лемме 8 справедлива оценка

$$
\left|\mathrm{E} \varphi\left(e^{\xi_{h_{1}}+\xi_{h_{2}}}\right)-\varphi(E)\right| \leqslant l(\varphi) \mathrm{E}\left\|e^{\xi_{h_{1}}+\xi_{h_{2}}}-E\right\| \leqslant l(\varphi) O\left(h^{1 / 2}\right) .
$$

Аналогичным образом $\left|\varphi(E)-\mathrm{E} \varphi\left(e^{\xi_{h}}\right)\right| \leqslant l(\varphi) O\left(h^{1 / 2}\right)$. Лемма доказана. 
СлЕДСТВИЕ 3. Пусть выполнены условия леммы 9, и пусть $\mu_{1}, \mu_{2}-$ случайные величины, принимающие значения в группе $G$, такие, что $\mu_{1}, \mu_{2}, \xi_{h_{1}}, \xi_{h_{2}}$ - независимые случайные величины и $\mu_{1}, \mu_{2}, \xi_{h}$ - независимые случайные величины. Тогда

$$
\left|\mathrm{E} \varphi\left(\mu_{1} e^{\xi_{h_{1}}} e^{\xi_{h_{2}}} \mu_{2}\right)-\mathrm{E} \varphi\left(\mu_{1} e^{\xi_{h}} \mu_{2}\right)\right| \leqslant l(\varphi) O\left(h^{1 / 2}\right),
$$

где константа в $O\left(h^{1 / 2}\right)$ зависит только от группь $G$.

Лемма 10. Пусть случайные величины $\xi_{h_{1}}, \ldots, \xi_{h_{n}}$ независимы. Тогда

$$
\begin{aligned}
& \left\|\mathrm{E}\left(e^{\xi_{h_{1}}+\cdots+\xi_{h_{n}}}-e^{\xi_{h_{1}}+\cdots+\xi_{h_{k}}} e^{\xi_{h_{k+1}}+\cdots+\xi_{h_{n}}}\right)\right\| \leqslant O\left(h^{3 / 2}\right), \\
& \mathrm{E}\left\|e^{\xi_{h_{1}}+\cdots+\xi_{h_{n}}}-e^{\xi_{h_{1}}+\cdots+\xi_{h_{k}}} e^{\xi_{h_{k+1}}+\cdots+\xi_{h_{n}}}\right\|^{2} \leqslant O\left(h^{2}\right), \quad h \rightarrow 0,
\end{aligned}
$$

где $h=h_{1}+\cdots+h_{n} ;$ константы в $O\left(h^{3 / 2}\right), O\left(h^{2}\right)$ зависят только от группь $G$.

ДокАЗАТЕЛЬСТво. Пусть $\eta_{1}=\xi_{h_{1}}+\cdots+\xi_{h_{k}}$ и $\eta_{2}=\xi_{h_{k+1}}+\cdots+\xi_{h_{n}}$. Тогда

$$
\begin{aligned}
e^{\eta_{1}+\eta_{2}}-e^{\eta_{1}} e^{\eta_{2}}= & \frac{\eta_{2} \eta_{1}-\eta_{1} \eta_{2}}{2}-e^{\eta_{1}} \sum_{i=3}^{\infty} \frac{\left(\eta_{2}\right)^{i}}{i !}-\sum_{i=3}^{\infty} \frac{\left(\eta_{1}\right)^{i}}{i !} e^{\eta_{2}} \\
& -\frac{\left(\eta_{1}\right)^{2}}{2 !} \eta_{2}-\eta_{1} \frac{\left(\eta_{2}\right)^{2}}{2 !}-\frac{\left(\eta_{1}\right)^{2}}{2 !} \frac{\left(\eta_{2}\right)^{2}}{2 !}+\sum_{i=3}^{\infty} \frac{\left(\eta_{1}+\eta_{2}\right)^{i}}{i !}
\end{aligned}
$$

Поскольку $\mathrm{E} \eta_{1}=\mathrm{E} \eta_{2}=0$ и $\eta_{1}, \eta_{2}$ независимы, то

$$
\begin{aligned}
\mathrm{E}\left(e^{\eta_{1}+\eta_{2}}-e^{\eta_{1}} e^{\eta_{2}}\right)= & -\mathrm{E}\left(e^{\eta_{1}} \sum_{i=3}^{\infty} \frac{\left(\eta_{2}\right)^{i}}{i !}\right)-\mathrm{E}\left(\sum_{i=3}^{\infty} \frac{\left(\eta_{1}\right)^{i}}{i !} e^{\eta_{2}}\right) \\
& -\mathrm{E}\left(\frac{\left(\eta_{1}\right)^{2}}{2 !} \frac{\left(\eta_{2}\right)^{2}}{2 !}\right)+\mathrm{E} \sum_{i=3}^{\infty} \frac{\left(\eta_{1}+\eta_{2}\right)^{i}}{i !}
\end{aligned}
$$

Из лемм 7 и 8 следует, что

$$
\begin{aligned}
& \left\|\mathrm{E}\left(e^{\eta_{1}+\eta_{2}}-e^{\eta_{1}} e^{\eta_{2}}\right)\right\| \\
& \quad \leqslant d \mathrm{E} \sum_{i=3}^{\infty} \frac{\left\|\eta_{2}\right\|^{i}}{i !}+d \mathrm{E} \sum_{i=3}^{\infty} \frac{\left\|\eta_{1}\right\|^{i}}{i !}+\mathrm{E} \frac{\left\|\eta_{1}\right\|^{2}}{2 !} \mathrm{E} \frac{\left\|\eta_{2}\right\|^{2}}{2 !}+\mathrm{E} \sum_{i=3}^{\infty} \frac{\left\|\eta_{1}+\eta_{2}\right\|^{i}}{i !} \leqslant O\left(h^{3 / 2}\right) .
\end{aligned}
$$

Здесь $d=\max _{X \in G}\|X\|$. Таким образом, первое утверждение леммы доказано. Из равенства (2.6) получаем, что

$$
\begin{gathered}
\left\|e^{\eta_{1}+\eta_{2}}-e^{\eta_{1}} e^{\eta_{2}}\right\|^{2} \leqslant 7\left(\left\|\frac{\eta_{2} \eta_{1}-\eta_{1} \eta_{2}}{2}\right\|^{2}+\left\|e^{\eta_{1}} \sum_{i=3}^{\infty} \frac{\left(\eta_{2}\right)^{i}}{i !}\right\|^{2}+\left\|\sum_{i=3}^{\infty} \frac{\left(\eta_{1}\right)^{i}}{i !} e^{\eta_{2}}\right\|^{2}\right. \\
\left.+\left\|\frac{\left(\eta_{1}\right)^{2}}{2 !} \eta_{2}\right\|^{2}+\left\|\eta_{1} \frac{\left(\eta_{2}\right)^{2}}{2 !}\right\|^{2}+\left\|\frac{\left(\eta_{1}\right)^{2}}{2 !} \frac{\left(\eta_{2}\right)^{2}}{2 !}\right\|^{2}+\left\|\sum_{i=3}^{\infty} \frac{\left(\eta_{1}+\eta_{2}\right)^{i}}{i !}\right\|^{2}\right) .
\end{gathered}
$$


Далее опять применяем леммы 7 и 8:

$$
\begin{aligned}
& \mathrm{E}\left\|e^{\eta_{1}+\eta_{2}}-e^{\eta_{1}} e^{\eta_{2}}\right\|^{2} \\
& \qquad \begin{aligned}
& 7\left(\mathrm{E}\left\|\eta_{1}\right\|^{2} \mathrm{E}\left\|\eta_{2}\right\|^{2}+d^{2} \mathrm{E}\left\|\sum_{i=3}^{\infty} \frac{\left(\eta_{2}\right)^{i}}{i !}\right\|^{2}+d^{2} \mathrm{E}\left\|\sum_{i=3}^{\infty} \frac{\left(\eta_{1}\right)^{i}}{i !}\right\|^{2}\right. \\
& \left.+\frac{\mathrm{E}\left\|\eta_{1}\right\|^{4}}{4} \mathrm{E}\left\|\eta_{2}\right\|^{2}+\mathrm{E}\left\|\eta_{1}\right\|^{2} \frac{\mathrm{E}\left\|\eta_{2}\right\|^{4}}{4}+\frac{\mathrm{E}\left\|\eta_{1}\right\|^{4}}{4} \frac{\mathrm{E}\left\|\eta_{2}\right\|^{4}}{4}+\mathrm{E}\left\|\sum_{i=3}^{\infty} \frac{\left(\eta_{1}+\eta_{2}\right)^{i}}{i !}\right\|^{2}\right) \\
& \leqslant O\left(h^{2}\right) \quad(h \rightarrow 0) .
\end{aligned}
\end{aligned}
$$

Лемма доказана.

ЛЕМма 11. Пусть $\varphi \in C^{2}(M), \xi_{h_{1}}, \ldots, \xi_{h_{n}}$ - независимые случайные величинь с плотностями $p_{h_{1}}, \ldots, p_{h_{n}}$. Тогда

$$
\left|\mathrm{E} \varphi\left(e^{\xi_{h_{1}}+\cdots+\xi_{h_{k}}} e^{\xi_{h_{k+1}}+\cdots+\xi_{h_{n}}}\right)-\mathrm{E} \varphi\left(e^{\xi_{h_{1}}+\cdots+\xi_{h_{n}}}\right)\right| \leqslant m(\varphi) O\left(h^{3 / 2}\right) \quad(h \rightarrow 0),
$$

$2 \partial e$

$$
\begin{gathered}
m(\varphi)=\max _{X \in B}\left\{\left\|\varphi^{\prime}(X)\right\|,\left\|\varphi^{\prime \prime}(X)\right\|\right\} \\
\left\|\varphi^{\prime}(X)\right\|=\max _{Y \in M} \frac{\left|\varphi^{\prime}(X) Y\right|}{\|Y\|}, \quad\left\|\varphi^{\prime \prime}(X)\right\|=\max _{Y, Z \in M} \frac{\left|\varphi^{\prime \prime}(X)(Y, Z)\right|}{\|Y\|\|Z\|},
\end{gathered}
$$

$h=h_{1}+\cdots+h_{n}, B-$ шар в $M$ с иентром в нуле, содержащий $G$, константа в $O\left(h^{3 / 2}\right)$ зависит только от группь $G$.

ДокАЗАТЕЛЬСтво. Пусть $\eta_{1}=\xi_{h_{1}}+\cdots+\xi_{h_{k}}$ и $\eta_{2}=\xi_{h_{k+1}}+\cdots+\xi_{h_{n}}$. По формуле Тейлора с остаточньм членом в форме Лагранжа

$$
\begin{aligned}
\varphi\left(e^{\eta_{1}} e^{\eta_{2}}\right)-\varphi\left(e^{\eta_{1}+\eta_{2}}\right)= & \varphi^{\prime}\left(e^{\eta_{1}+\eta_{2}}\right)\left(e^{\eta_{1}} e^{\eta_{2}}-e^{\eta_{1}+\eta_{2}}\right) \\
& +\frac{1}{2} \varphi^{\prime \prime}(\zeta)\left(e^{\eta_{1}} e^{\eta_{2}}-e^{\eta_{1}+\eta_{2}}, e^{\eta_{1}} e^{\eta_{2}}-e^{\eta_{1}+\eta_{2}}\right),
\end{aligned}
$$

где $\zeta$ - точка, лежащая на отрезке $\left[e^{\eta_{1}} e^{\eta_{2}}, e^{\eta_{1}+\eta_{2}}\right]$. Тогда

$$
\begin{aligned}
& \left|\mathrm{E} \varphi\left(e^{\eta_{1}} e^{\eta_{2}}\right)-\mathrm{E} \varphi\left(e^{\eta_{1}+\eta_{2}}\right)\right| \leqslant\left|\varphi^{\prime}(E) \mathrm{E}\left(e^{\eta_{1}} e^{\eta_{2}}-e^{\eta_{1}+\eta_{2}}\right)\right| \\
& \quad+\left|\mathrm{E}\left(\left(\varphi^{\prime}\left(e^{\eta_{1}+\eta_{2}}\right)-\varphi^{\prime}(E)\right)\left(e^{\eta_{1}} e^{\eta_{2}}-e^{\eta_{1}+\eta_{2}}\right)\right)\right|+\frac{1}{2} m(\varphi) \mathrm{E}\left\|e^{\eta_{1}} e^{\eta_{2}}-e^{\eta_{1}+\eta_{2}}\right\|^{2}
\end{aligned}
$$

Будем оценивать каждое слагаемое в предыдущем неравенстве. По лемме 10

$$
\left|\varphi^{\prime}(E) \mathrm{E}\left(e^{\eta_{1}} e^{\eta_{2}}-e^{\eta_{1}+\eta_{2}}\right)\right| \leqslant m(\varphi)\left\|\mathrm{E}\left(e^{\eta_{1}} e^{\eta_{2}}-e^{\eta_{1}+\eta_{2}}\right)\right\|=m(\varphi) O\left(h^{3 / 2}\right) .
$$

По формуле Лагранжа

$$
\left(\varphi^{\prime}\left(e^{\eta_{1}+\eta_{2}}\right)-\varphi^{\prime}(E)\right)\left(e^{\eta_{1}} e^{\eta_{2}}-e^{\eta_{1}+\eta_{2}}\right)=\varphi^{\prime \prime}(\theta)\left(e^{\eta_{1}+\eta_{2}}-E, e^{\eta_{1}} e^{\eta_{2}}-e^{\eta_{1}+\eta_{2}}\right)
$$


где $\theta$ - точка, лежащая на отрезке $\left[e^{\eta_{1}+\eta_{2}}, E\right]$; следовательно,

$$
\mathrm{E}\left|\left(\varphi^{\prime}\left(e^{\eta_{1}+\eta_{2}}\right)-\varphi^{\prime}(E)\right)\left(e^{\eta_{1}} e^{\eta_{2}}-e^{\eta_{1}+\eta_{2}}\right)\right| \leqslant m(\varphi) \mathrm{E}\left(\left\|e^{\eta_{1}+\eta_{2}}-E\right\|\left\|e^{\eta_{1}} e^{\eta_{2}}-e^{\eta_{1}+\eta_{2}}\right\|\right) .
$$

Согласно неравенству Коши-Буняковского и леммам 8 и 10

$$
\begin{aligned}
& m(\varphi) \mathrm{E}\left(\left\|e^{\eta_{1}+\eta_{2}}-E\right\|\left\|e^{\eta_{1}} e^{\eta_{2}}-e^{\eta_{1}+\eta_{2}}\right\|\right) \\
& \quad \leqslant m(\varphi)\left(\mathrm{E}\left\|e^{\eta_{1}+\eta_{2}}-E\right\|^{2}\right)^{1 / 2}\left(\mathrm{E}\left\|e^{\eta_{1}} e^{\eta_{2}}-e^{\eta_{1}+\eta_{2}}\right\|^{2}\right)^{1 / 2} \leqslant m(\varphi) O\left(h^{3 / 2}\right) .
\end{aligned}
$$

По лемме 10

$$
\mathrm{E}\left\|e^{\eta_{1}} e^{\eta_{2}}-e^{\eta_{1}+\eta_{2}}\right\|^{2} \leqslant O\left(h^{2}\right) .
$$

Подставляя в (2.7) оценки (2.8)-(2.10), получаем утверждение леммы.

СЛЕДСТВИЕ 4. Пусть выполнены условия леммы 11, и пусть $\mu_{1}, \mu_{2}-$ случайные величины, принимающие значения в группе $G$, такие, что $\mu_{1}, \mu_{2}, \xi_{h_{1}}, \ldots, \xi_{h_{n}}-$ независимые случайные величины. Тогда

$$
\begin{aligned}
& \left|\mathrm{E} \varphi\left(\mu_{1} e^{\xi_{h_{1}}+\cdots+\xi_{h_{k}}} e^{\xi_{h_{k+1}}+\cdots+\xi_{h_{k+l}}} e^{\xi_{h_{k+l+1}}+\cdots+\xi_{h_{n}}} \mu_{2}\right)-\mathrm{E} \varphi\left(\mu_{1} e^{\xi_{h_{1}}+\cdots+\xi_{h_{n}}} \mu_{2}\right)\right| \\
& \quad \leqslant m(\varphi) O\left(h^{3 / 2}\right)
\end{aligned}
$$

где константа в $O\left(h^{3 / 2}\right)$ зависит только от группь $G$.

ТЕОРема 2. Пусть $\varphi \in C^{2}(M), \xi_{h_{1}}, \ldots, \xi_{h_{n}}$ - независимые случайные величины с плотностями $p_{h_{1}}, \ldots, p_{h_{n}}$. Тогда

$$
\left|\mathrm{E} \varphi\left(e^{\xi_{h_{1}}} \ldots e^{\xi_{h_{n}}}\right)-\mathrm{E} \varphi\left(e^{\xi_{h_{1}}+\cdots+\xi_{h_{n}}}\right)\right| \leqslant m(\varphi) O\left(h^{3 / 2}\right),
$$

где $h=h_{1}+\cdots+h_{n}, m(\varphi)$ определено в лемме $11, B$ - шар в $M$ с иентром в нуле, содержащий $G$, константа в $O\left(h^{3 / 2}\right)$ зависит только от әруппы $G$.

ДокАЗАТЕЛЬСтво. Пусть $t_{0}=0, t_{1}=h_{1}, t_{2}=h_{1}+h_{2}, \ldots, t_{n}=h$. Для произвольных $q \in \mathbb{N}$ и $l \in \mathbb{N}, l \leqslant 2^{q}$, введем следующие обозначения:

$$
\xi_{l}^{q} \stackrel{\text { def }}{=} \xi_{h_{i+1}}+\xi_{h_{i+2}}+\cdots+\xi_{h_{i+j-1}}, \quad \widetilde{\xi}_{l}^{q} \stackrel{\text { def }}{=} \begin{cases}\xi_{h_{i+j}}, & t_{i+j-1}<\frac{l h}{2^{q}} \text { и } j>0, \\ 0, & t_{i+j-1}=\frac{l h}{2^{q}} \text { или } j=0,\end{cases}
$$

где $t_{i-1}<(l-1) h / 2^{q}, t_{i}, t_{i+1}, \ldots, t_{i+j-1} \in\left[(l-1) h / 2^{q}, l h / 2^{q}\right], t_{i+j}>l h / 2^{q}$. Заметим, что

$$
\begin{aligned}
& \xi_{l}^{q-1}= \begin{cases}\xi_{2 l-1}^{q}, & t_{i} \notin\left[\frac{(2 l-1) h}{2^{q}}, \frac{l h}{2^{q-1}}\right], i=0, \ldots, n, \\
\xi_{2 l-1}^{q}+\widetilde{\xi}_{2 l-1}^{q}+\xi_{2 l}^{q}, & \text { в противном случае, }\end{cases} \\
& \widetilde{\xi}_{l}^{q-1}=\left\{\begin{array}{l}
\widetilde{\xi}_{2 l-1}^{q}, \quad t_{i} \notin\left[\frac{(2 l-1) h}{2^{q}}, \frac{l h}{2^{q-1}}\right], i=0, \ldots, n, \\
\widetilde{\xi}_{2 l}^{q}, \quad \text { в противном случае },
\end{array}\right. \\
& \xi_{1}^{q}+\widetilde{\xi}_{1}^{q}+\cdots+\xi_{2^{q}-1}^{q}+\widetilde{\xi}_{2^{q}-1}^{q}+\xi_{2^{q}}^{q}=\xi_{h_{1}}+\cdots+\xi_{h_{n}} .
\end{aligned}
$$


Учитьвая (2.11)-(2.13) и следствие 4, получаем

$$
\begin{aligned}
& \mid \mathrm{E} \varphi\left(e^{\xi_{1}^{q}} e^{\widetilde{\xi}_{1}^{q}} e^{\xi_{2}^{q}} e^{\widetilde{\xi}_{2}^{q}} \cdots e^{\xi_{2 l-1}^{q}} e^{\widetilde{\xi}_{2 l-1}^{q}} e^{\xi_{2 l}^{q}} e^{\widetilde{\xi}_{2 l}^{q}} \cdots e^{\xi_{2 q}^{q}-1} e^{\widetilde{\xi}_{2 q}^{q}-1} e^{\xi_{2}^{q}}\right) \\
& -\mathrm{E} \varphi\left(e^{\xi_{1}^{q-1}} e^{\tilde{\xi}_{1}^{q-1}} \cdots e^{\xi_{l}^{q-1}} \cdots e^{\xi_{2 q-1}^{q-1}}\right) \\
& \leqslant \mid \mathrm{E} \varphi\left(e^{\xi_{1}^{q}} e^{\tilde{\xi}_{1}^{q}} e^{\xi_{2}^{q}} e^{\tilde{\xi}_{2}^{q}} \cdots e^{\xi_{2 l-1}^{q}} e^{\widetilde{\xi}_{2 l-1}^{q}} e^{\xi_{2 l}^{q}} e^{\tilde{\xi}_{2 l}^{q}} \cdots e^{\xi_{2}^{q}-1} e^{\widetilde{\xi}_{2}^{q}-1} e^{\xi_{2 q}^{q}}\right) \\
& -\mathrm{E} \varphi\left(e^{\xi_{1}^{q-1}} e^{\widetilde{\xi}_{1}^{q-1}} \cdots e^{\xi_{2 l-1}^{q}} e^{\tilde{\xi}_{2 l-1}^{q}} e^{\xi_{2 l}^{q}} e^{\tilde{\xi}_{2 l}^{q}} \cdots e^{\xi_{2 q}^{q}-1} e^{\widetilde{\xi}_{2 q}^{q}-1} e^{\xi_{2 q}^{q}}\right) \mid \\
& +\mid \mathrm{E} \varphi\left(e^{\xi_{1}^{q-1}} e^{\tilde{\xi}_{1}^{q-1}} \cdots e^{\xi_{l}^{q-1}} e^{\tilde{\xi}_{l}^{q-1}} \cdots e^{\xi_{2}^{q}-1} e^{\tilde{\xi}_{2 q}^{q}-1} e^{\xi_{2}^{q}}\right) \\
& -\mathrm{E} \varphi\left(e^{\xi_{1}^{q-1}} e^{\tilde{\xi}_{1}^{q-1}} \cdots e^{\xi_{l}^{q-1}} e^{\widetilde{\xi}_{l}^{q-1}} \cdots e^{\xi_{2}^{q-1}}\right) \\
& =2^{q} O\left(\left(\frac{h}{2^{q-1}}\right)^{3 / 2}\right) \text {. }
\end{aligned}
$$

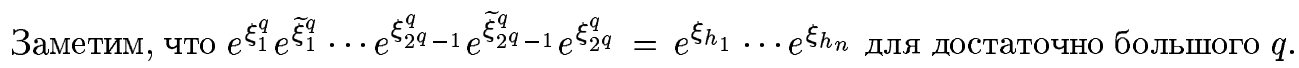
Поэтому, учитьвая (2.14), получаем

$$
\begin{aligned}
& \left|\mathrm{E} \varphi\left(e^{\xi_{h_{1}}} \ldots e^{\xi_{h_{n}}}\right)-\mathrm{E} \varphi\left(e^{\xi_{h_{1}}+\cdots+\xi_{h_{n}}}\right)\right| \\
& \quad \leqslant\left|\mathrm{E} \varphi\left(e^{\xi_{1}^{q}} e^{\tilde{\xi}_{1}^{q}} \cdots e^{\xi_{2}^{q}}\right)-\mathrm{E} \varphi\left(e^{\xi_{1}^{q-1}} e^{\tilde{\xi}_{1}^{q-1}} \cdots e^{\xi_{2 q-1}^{q-1}}\right)\right|+\cdots \\
& \quad+\left|\mathrm{E} \varphi\left(e^{\xi_{1}^{1}} e^{\tilde{\xi}_{1}^{1}} e^{\xi_{2}^{1}}\right)-\mathrm{E} \varphi\left(e^{\xi_{h_{1}}+\cdots+\xi_{h_{n}}}\right)\right| \\
& \quad \leqslant \sum_{s=0}^{q} 2^{s} O\left(\left(\frac{h}{2^{s-1}}\right)^{3 / 2}\right) \leqslant O\left(h^{3 / 2}\right) \sum_{s=0}^{\infty} 2^{(3-s) / 2}=O\left(h^{3 / 2}\right) .
\end{aligned}
$$

Теорема доказана.

В дальнейшем нам потребуется следующее небольшое усиление теоремы 2.

СлЕДСТВИЕ 5. Пусть выполнены условия теоремы 2, и пусть $\mu_{1}, \mu_{2}-$ случайные величины, принимающие значения в группе $G$, такие, что случайные величины $\mu_{1}, \mu_{2}, \xi_{h_{1}}, \ldots, \xi_{h_{n}}-$ независимы. Тогда

$$
\left|\mathrm{E} \varphi\left(\mu_{1} e^{\xi_{h_{1}}} \ldots e^{\xi_{h_{n}}} \mu_{2}\right)-\mathrm{E} \varphi\left(\mu_{1} e^{\xi_{h_{1}}+\cdots+\xi_{h_{n}}} \mu_{2}\right)\right|=m(\varphi) O\left(h^{3 / 2}\right) ;
$$

константа в $O\left(h^{3 / 2}\right)$ зависит только от группь $G$.

3. Основные результаты. Пусть $T=\left\{0=t_{0}<t_{1}<\cdots<t_{n}=t\right\}$ - разбиение отрезка $[0, t]$. Определим оператор $A_{t}^{T}: C(G) \rightarrow C(G)$ следуюшим соотношением:

$$
\left(A_{t}^{T} \varphi\right)(X)=\mathrm{E} \varphi\left(e^{\xi_{h_{1}}} \cdots e^{\xi_{h_{n}}} X\right)
$$

где $h_{1}=t_{1}-t_{0}, \ldots, h_{n}=t_{n}-t_{n-1}-$ длины отрезков разбиения $T$.

ЗАМЕЧАНИЕ. Согласно следствию 1

$$
\begin{aligned}
\left(A_{t}^{T} \varphi\right)(X)= & \int_{G} \cdots \int_{G} \varphi(Y X) p\left(Y, Y_{1}, t_{1}\right) \\
& \times p\left(Y_{1}, Y_{2}, t_{2}-t_{1}\right) \cdots p\left(Y_{n-1}, E, t_{n}-t_{n-1}\right) d Y_{1} \cdots d Y_{n-1} .
\end{aligned}
$$

Из определения оператора $A_{t}^{T}$ непосредственно следует 
ЛЕмма 12. Пусть $T$ - произвольное разбиение отрезка $[0, t] u \varphi \in C(G)$. Тогда $\left\|A_{t}^{T}\right\|=1$. Кроме того, если $Y \in G$ таково, что $|\varphi(X Y)-\varphi(X)| \leqslant \varepsilon$ дл всех $X \in G$, mo $\left|\left(A_{t}^{T} \varphi\right)(X Y)-\left(A_{t}^{T} \varphi\right)(X)\right| \leqslant \varepsilon$ для всех $X \in G$.

ЛЕМма 13. Пусть имеется последовательность разбиений $\left\{\widetilde{T}_{n}\right\}$ отрезка $[0, t]$ с диаметрами разбиений $\Delta \widetilde{T}_{n} \rightarrow 0$. Тогда из последовательности $\left\{\widetilde{T}_{n}\right\}$ можно выделить подпоследовательность $\left\{T_{n}\right\}$ так, чтобы для произвольной функиии $\varphi \in C(G)$ последовательность $\left\{A_{t}^{T_{n}} \varphi\right\}$ была фундаментальной в $C(G)$.

ДокАЗАТЕЛЬСТво. Для любой функции $\varphi \in C(G)$ по лемме 12 и теореме Арцела-Асколи из последовательности $A_{t}^{\widetilde{T}_{n}} \varphi$ можно выделить фундаментальную в $C(G)$ подпоследовательность. С помощью диагональной процедуры можно выбрать подпоследовательность $T_{n}$ последовательности $\left\{\widetilde{T}_{n}\right\}$ такую, что последовательность $\left\{A_{t}^{T_{n}} \varphi\right\}$ фундаментальна в $C(G)$ для всех $\varphi$ из некоторого счетного плотного в $C(G)$ множества. В силу равностепенной непрерьвности семейства операторов $\left\{A_{t}^{T_{n}}: n \in \mathbb{N}\right\}$ $\left(\left\|A_{t}^{T_{n}}\right\|=1\right)$ последовательность $\left\{A_{t}^{T_{n}} \varphi\right\}$ фундаментальна в $C(G)$ для любой $\varphi \in C(G)$. Лемма доказана.

Возьмем последовательность разбиений $\left\{k t / 2^{n}: k=0, \ldots, 2^{n}\right\}$ отрезка $[0, t]$. Согласно лемме 13 из нее можно выделить подпоследовательность разбиений $T_{n}$ так, чтобы для любой функции $\varphi \in C(G)$ последовательность $\left\{A_{t}^{T_{n}} \varphi\right\}$ была фундаментальной в $C(G)$. Следовательно, существует линейный оператор $A_{t}: C(G) \rightarrow C(G)$ такой, что для любой функции $\varphi \in C(G)$ последовательность $\left\{A_{t}^{T_{n}} \varphi\right\}$ сходится к $A_{t} \varphi$ в пространстве $C(G)$. В силу леммы $12\left\|A_{t}\right\| \leqslant 1$.

Teоpema 3. Пусть $\varphi \in C(G)$. Тогда

$$
\begin{aligned}
\left(A_{t} \varphi\right)(X)= & \lim _{\max _{i} \rightarrow 0} \int_{G} \cdots \int_{G} \varphi\left(X_{1}\right) p\left(X_{1}, X_{2}, h_{1}\right) \\
& \quad \times p\left(X_{2}, X_{3}, h_{2}\right) \cdots p\left(X_{n}, X, h_{n}\right) d X_{1} \cdots d X_{n} .
\end{aligned}
$$

ДокАЗАТЕЛЬСтво. Сначала мы докажем эту формулу для $\varphi \in C^{2}(B)$, где $B$ - шар в $M$ с центром в нуле, содержащий $G$. Фиксируем $\varepsilon>0$ и докажем, что можно выбрать $\delta>0$ так, чтобы $\left|\left(A_{t} \varphi\right)(X)-\left(A_{t}^{T} \varphi\right)(X)\right| \leqslant \varepsilon$ для любого разбиения $T$ с диаметром $\Delta T$ меньшим $\delta$. Выберем $K_{0} \in \mathbb{N}$ так, чтобы для любого $k \geqslant K_{0}$

$$
\left\|\left(A_{t}^{T_{k}} \varphi\right)-\left(A_{t} \varphi\right)\right\|<\frac{\varepsilon}{3}
$$

Пусть $K \geqslant K_{0}, L$ - число отрезков разбиения $T_{K}$. Для произвольного разбиения $T$ отрезка $[0, t]$ с $\Delta T<h / L$ обозначим $\widetilde{T}=T \cup T_{K}$. Пусть $h_{1}, \ldots, h_{s}$ - отрезки разбиения $\widetilde{T}$; тогда $h_{1}, \ldots, h_{i_{1}}+h_{i_{1}+1}, \ldots, h_{i_{L-1}}+h_{i_{L-1}+1}, \ldots, h_{s}$ - отрезки разбиения $T_{0}$. Для следующей оценки фиксируем разбиение $T_{k}, k>K$. Очевидно,

$$
\left\|\left(A_{t} \varphi\right)-\left(A_{t}^{T} \varphi\right)\right\| \leqslant\left\|\left(A_{t} \varphi\right)-\left(A_{t}^{T_{k}} \varphi\right)\right\|+\left\|\left(A_{t}^{T_{k}} \varphi\right)-\left(A_{t}^{\widetilde{T}} \varphi\right)\right\|+\left\|\left(A_{t}^{\widetilde{T}} \varphi\right)-\left(A_{t}^{T} \varphi\right)\right\| .
$$


По следствию 3

$$
\begin{aligned}
& \left\|\left(A_{t}^{\widetilde{T}} \varphi\right)-\left(A_{t}^{T} \varphi\right)\right\| \\
& \leqslant\left\|\mathrm{E} \varphi\left(e^{\xi_{h_{1}}} \cdots e^{\xi_{h_{s}}} X\right)-\mathrm{E} \varphi\left(e^{\xi_{h_{1}}} \cdots e^{\xi_{h_{i_{1}}+h_{i_{1}+1}}} \cdots e^{\xi_{h_{i_{L-1}}}} e^{\xi_{h_{i_{L-1}}+1}} \cdots e^{\xi_{h_{s}} X}\right)\right\|+\cdots
\end{aligned}
$$

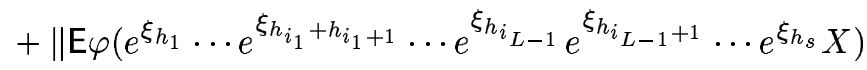

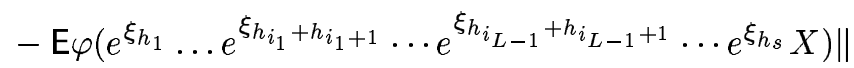

$$
\begin{aligned}
& \leqslant l(\varphi) O(\sqrt{\Delta T})(L-1) \text {. }
\end{aligned}
$$

Пусть $n L-$ число отрезков разбиения $T_{k}$. Заметим, что

$$
\begin{aligned}
& \left\|\left(A_{t}^{T_{k}} \varphi\right)-\left(A_{t}^{\widetilde{T}} \varphi\right)\right\| \\
& =\left\|\mathrm{E} \varphi\left(\left(e^{\xi_{1}^{1}} \cdots e^{\xi_{n}^{1}}\right) \cdots\left(e^{\xi_{1}^{L}} \cdots e^{\xi_{n}^{L}}\right) X\right)-\mathrm{E} \varphi\left(\left(e^{\xi_{h_{1}}} \cdots e^{\xi_{h_{1}}}\right) \cdots\left(e^{\xi_{h_{j_{n-1}+1}}} \cdots e^{\xi_{h_{s}}}\right) X\right)\right\| \\
& \leqslant\left\|\mathrm{E} \varphi\left(\left(e^{\xi_{1}^{1}} \cdots e^{\xi_{n}^{1}}\right) \cdots\left(e^{\xi_{1}^{L}} \cdots e^{\xi_{n}^{L}}\right) X\right)-\mathrm{E} \varphi\left(\left(e^{\xi_{1}^{1}+\cdots+\xi_{n}^{1}}\right) \cdots\left(e^{\xi_{1}^{L}+\cdots+\xi_{n}^{L}}\right) X\right)\right\| \\
& \leqslant\left\|\mathrm{E} \varphi\left(\left(e^{\xi_{1}^{1}+\cdots+\xi_{n}^{1}}\right) \cdots\left(e^{\xi_{1}^{L}+\cdots+\xi_{n}^{L}}\right) X\right)-\mathrm{E} \varphi\left(e^{\zeta_{r}^{1}} \cdots e^{\zeta_{r}^{L}} X\right)\right\| \\
& +\left\|\mathrm{E} \varphi\left(e^{\zeta_{r}^{1}} \cdots e^{\zeta_{r}^{L}} X\right)-\mathrm{E} \varphi\left(\left(e^{\xi_{h_{1}}+\cdots+\xi_{h_{j_{1}}}}\right) \cdots\left(e^{\xi_{h_{j_{n-1}+1}}+\cdots+\xi_{h_{s}}}\right) X\right)\right\| \\
& +\| \mathrm{E} \varphi\left(\left(e^{\xi_{h_{1}}+\cdots+\xi_{h_{j_{1}}}}\right) \cdots\left(e^{\xi_{h_{n-1}+1}+\cdots+\xi_{h_{s}}}\right) X\right) \\
& -\mathrm{E} \varphi\left(\left(e^{\xi_{h_{1}}} \cdots e^{\xi_{h_{j_{1}}}}\right) \cdots\left(e^{\xi_{h_{j_{n-1}}+1}} \cdots e^{\xi_{h_{s}}}\right) X\right) \| .
\end{aligned}
$$

В предыдущем выражении $h_{1}+\cdots+h_{j_{1}}=t / L, h_{j_{1}+1}+\cdots+h_{j_{2}}=t / L, \ldots, h_{j_{n-1}+1}+\cdots$ $+h_{s}=t / L, \xi_{i}^{j}$ - независимые одинаково распределенные случайные величины со значениями в $T_{E} G$, имеюшие там плотность $p_{h}(X), h=t /(L n), \zeta_{r}^{1}, \ldots, \zeta_{r}^{L}$ - независимые одинаково распределенные гауссовские случайные величины с матрицей ковариаций $r E_{m}$. По следствию 5

$$
\begin{aligned}
& \left\|\mathrm{E} \varphi\left(\left(e^{\xi_{1}^{1}} \cdots e^{\xi_{n}^{1}}\right) \cdots\left(e^{\xi_{1}^{L}} \cdots e^{\xi_{n}^{L}}\right) X\right)-\mathrm{E} \varphi\left(\left(e^{\xi_{1}^{1}+\cdots+\xi_{n}^{1}}\right) \cdots\left(e^{\xi_{1}^{L}+\cdots+\xi_{n}^{L}}\right) X\right)\right\| \\
& \quad \leqslant\left\|\mathrm{E} \varphi\left(\left(e^{\xi_{1}^{1}} \cdots e^{\xi_{n}^{1}}\right) \cdots\left(e^{\xi_{1}^{L}} \cdots e^{\xi_{n}^{L}}\right) X\right)-\mathrm{E} \varphi\left(e^{\xi_{1}^{1}+\cdots+\xi_{n}^{1}} \cdots\left(e^{\xi_{1}^{L}} \cdots e^{\xi_{n}^{L}}\right) X\right)\right\|+\cdots \\
& \quad+\left\|\mathrm{E} \varphi\left(e^{\xi_{1}^{1}+\cdots+\xi_{n}^{1}} \cdots\left(e^{\xi_{1}^{L}} \cdots e^{\xi_{n}^{L}}\right) X\right)-\mathrm{E} \varphi\left(e^{\xi_{1}^{1}+\cdots+\xi_{n}^{1}} \cdots e^{\xi_{1}^{L}+\cdots+\xi_{n}^{L}} X\right)\right\| \\
& \quad \leqslant m(\varphi) O\left(\left(\frac{t}{L}\right)^{3 / 2}\right) L \quad\left(\frac{t}{L} \rightarrow 0\right)
\end{aligned}
$$

и аналогично

$$
\begin{aligned}
& \| \mathrm{E} \varphi\left(\left(e^{\xi_{h_{1}}+\cdots+\xi_{h}}\right) \cdots\left(e^{\xi_{h_{j_{n-1}+1}}+\cdots+\xi_{h_{s}}}\right) X\right) \\
& -\mathrm{E} \varphi\left(\left(e^{\xi_{h_{1}}} \cdots e^{\xi_{h_{j_{1}}}}\right) \cdots\left(e^{\xi_{h_{j_{n-1}}+1}} \cdots e^{\xi_{h_{s}}}\right) X\right) \| \\
& \leqslant m(\varphi) O\left(\left(\frac{t}{L}\right)^{3 / 2}\right) L \quad\left(\frac{t}{L} \rightarrow 0\right) \text {. }
\end{aligned}
$$


Далее по следствию 2

$$
\begin{aligned}
& \left\|\mathrm{E} \varphi\left(\left(e^{\xi_{1}^{1}+\cdots+\xi_{n}^{1}}\right) \cdots\left(e^{\xi_{1}^{L}+\cdots+\xi_{n}^{L}}\right) X\right)-\mathrm{E} \varphi\left(e^{\zeta_{r}^{1}} \cdots e^{\zeta_{r}^{L}} X\right)\right\| \\
& \quad \leqslant\left|\mathrm{E} \varphi\left(e^{\xi_{1}^{1}+\cdots+\xi_{n}^{1}} \cdots e^{\xi_{1}^{L}+\cdots+\xi_{n}^{L}} X\right)-\mathrm{E} \varphi\left(e^{\zeta_{r}^{1}} \cdots e^{\xi_{1}^{L}+\cdots+\xi_{n}^{L}} X\right)\right|+\cdots \\
& \quad+\left|\mathrm{E} \varphi\left(e^{\zeta_{r}^{1}} \cdots e^{\xi_{1}^{L}+\cdots+\xi_{n}^{L}} X\right)-\mathrm{E} \varphi\left(e^{\zeta_{r}^{1}} \cdots e^{\zeta_{r}^{L}} X\right)\right| \\
& \quad=o(1) L \quad\left(\frac{t}{L n} \rightarrow 0\right) .
\end{aligned}
$$

Аналогично предыдущей оценке

$$
\left\|\mathrm{E} \varphi\left(e^{\zeta_{r}^{1}} \cdots e^{\zeta_{r}^{L}} X\right)-\mathrm{E} \varphi\left(\left(e^{\xi_{h_{1}}+\cdots+\xi_{h_{1}}}\right) \cdots\left(e^{\xi_{h_{j_{n-1}+1}}+\cdots+\xi_{h_{s}}}\right) X\right)\right\|=o(1) L \quad(\Delta T \rightarrow 0) .
$$

Учитывая (3.6) и (3.7), выберем $K \geqslant K_{0}$ так, чтобы

$$
\begin{gathered}
\left\|\mathrm{E} \varphi\left(\left(e^{\xi_{1}^{1}} \cdots e^{\xi_{n}^{1}}\right) \cdots\left(e^{\xi_{1}^{L}} \cdots e^{\xi_{n}^{L}}\right) X\right)-\mathrm{E} \varphi\left(\left(e^{\xi_{1}^{1}+\cdots+\xi_{n}^{1}}\right) \cdots\left(e^{\xi_{1}^{L}+\cdots+\xi_{n}^{L}}\right) X\right)\right\| \leqslant \frac{\varepsilon}{12} \\
\| \mathrm{E} \varphi\left(\left(e^{\xi_{h_{1}}+\cdots+\xi_{h_{j_{1}}}}\right) \cdots\left(e^{\xi_{h_{j_{n-1}+1}}+\cdots+\xi_{h_{s}}}\right) X\right) \\
-\mathrm{E} \varphi\left(\left(e^{\left.\left.\xi_{h_{1}} \cdots e^{\xi_{h_{1}}}\right) \cdots\left(e^{\xi_{h_{j_{n-1}}+1}} \cdots e^{\xi_{h_{s}}}\right) X\right) \| \leqslant \frac{\varepsilon}{12}}\right.\right.
\end{gathered}
$$

В соответствии с (3.8) выберем $k>K$ так, чтобы

$$
\left\|\mathrm{E} \varphi\left(\left(e^{\xi_{1}^{1}+\cdots+\xi_{n}^{1}}\right) \cdots\left(e^{\xi_{1}^{L}+\cdots+\xi_{n}^{L}}\right) X\right)-\mathrm{E} \varphi\left(e^{\zeta_{r}^{1}} \cdots e^{\zeta_{r}^{L}} X\right)\right\| \leqslant \frac{\varepsilon}{12} .
$$

Тогда по (3.4) и (3.9) существует $\delta>0$ такое, что для произвольного разбиения $T$ отрезка $[0, t]$ с $\Delta T<\delta$

$$
\begin{gathered}
\left\|\left(A_{t}^{\widetilde{T}} \varphi\right)-\left(A_{t}^{T} \varphi\right)\right\| \leqslant \frac{\varepsilon}{3} \\
\left\|\mathrm{E} \varphi\left(e^{\zeta_{r}^{1}} \cdots e^{\zeta_{r}^{L}} X\right)-\mathrm{E} \varphi\left(\left(e^{\xi_{h_{1}}+\cdots+\xi_{h_{1}}}\right) \cdots\left(e^{\xi_{h_{j_{n-1}+1}}+\cdots+\xi_{h_{s}}}\right) X\right)\right\| \leqslant \frac{\varepsilon}{12} .
\end{gathered}
$$

Учитьвая (3.5), (3.10)-(3.12) и (3.14), получаем, что

$$
\left\|\left(A_{t}^{T_{k}} \varphi\right)-\left(A_{t}^{\widetilde{T}} \varphi\right)\right\| \leqslant \frac{\varepsilon}{3} .
$$

Из (3.3), (3.2), (3.13) и (3.15) следует утверждение теоремы для $\varphi \in C^{2}(B)$. Следует отметить, что любая функция из $C^{2}(G)$ продолжается до функции из $C^{2}(B)$ (см. [14, гл. 6]); следовательно, теорема верна для всех $\varphi \in C^{2}(G)$. Для произвольной $\varphi \in C(G)$ фиксируем $\varepsilon>0$ и выберем $\psi \in C^{2}(G)$ так, чтобы $\|\psi-\varphi\|_{C(G)} \leqslant \varepsilon / 3$. Возьмем теперь $\delta>0$ так, чтобы $\left\|A_{t} \varphi-A_{t}^{T} \varphi\right\| \leqslant \varepsilon / 3$ для любого разбиения $T$ отрезка $[0, t] \mathrm{c} \Delta T \leqslant \delta$. Тогда $\left\|A_{t} \varphi-A_{t}^{T} \varphi\right\| \leqslant\left\|A_{t} \varphi-A_{t} \psi\right\|+\left\|A_{t} \psi-A_{t}^{T} \psi\right\|+\left\|A_{t}^{T} \psi-A_{t}^{T} \varphi\right\| \leqslant \varepsilon$ при $\Delta T \leqslant \delta$. Теорема доказана.

Из формулы (3.1) следует, что $A_{t} A_{s}=A_{t+s}$, т.е. операторы $A_{t}: C(G) \rightarrow C(G)$ образуют полугруппу. Напомним, что $\left\|A_{t}\right\| \leqslant 1$; значит, полугруппа $\left\{A_{t}\right\}$ сжимающая. Пусть $P$ - инфинитезимальньй оператор полугруппы $\left\{A_{t}\right\}$. 
Tеорема 4. Сжимающая полугруппа $\left\{A_{t}\right\}$ сильно непрерывна на пространстве $C(G)$. Область определения $D_{P}$ оператора $P$ содержит $C^{2}(G)$, причем $P \varphi=\frac{1}{2} \Delta \varphi$ прu $\varphi \in C^{2}(G)$.

ДокаЗАТЕЛЬСтво. Докажем сначала второе утверждение теоремы. Возьмем произвольную функцию $\varphi \in C^{2}(G)$. Как уже было сказано, $\varphi$ можно продолжить до функции из $C^{2}(B)$, где $B$ - шар в $M$, содержаший $G$, поэтому мы можем считать, что $\varphi \in$ $C^{2}(B)$. Фиксируем произвольное $h>0$. Применяя теорему 2 и следствие 2 , выберем $\delta>0$ такое, что для любого разбиения $T$ отрезка $[0, h]$ с $\Delta T<\delta$ и для любого $X \in G$

$$
\left|\mathrm{E} \varphi\left(e^{\xi_{h_{1}}+\cdots+\xi_{h_{s}}} X\right)-\mathrm{E} \varphi\left(e^{\zeta_{h}} X\right)\right|<h^{3 / 2}, \quad\left|\left(A_{h}^{T} \varphi\right)(X)-\left(A_{h} \varphi\right)(X)\right|<h^{3 / 2},
$$

где $h_{1}+\cdots+h_{s}=h, h_{1}, \ldots, h_{s}$ - отрезки разбиения $T, \zeta_{h}$ - гауссовская случайная величина с матрицей ковариаций $h E_{m}$. Согласно теореме 1

$$
\left|\left(A_{h}^{T} \varphi\right)(X)-\mathrm{E} \varphi\left(e^{\xi_{h_{1}}+\cdots+\xi_{h_{s}}}\right)\right| \leqslant m(\varphi) O\left(h^{3 / 2}\right) .
$$

Таким образом, учитывая $(3.16),(3.17)$ и равенство $A_{0} \varphi=\varphi$, имеем

$$
\begin{gathered}
(P \varphi)(X)=\frac{\left(A_{h} \varphi\right)(X)-\left(A_{0} \varphi\right)(X)}{h}=\frac{1}{h}\left(\mathrm{E} \varphi\left(e^{\zeta_{h}} X\right)-\varphi(X)\right)+O\left(h^{1 / 2}\right), \\
\mathrm{E} \varphi\left(e^{\zeta_{h}} X\right)=\frac{1}{(\sqrt{2 \pi h})^{m}} \int_{T_{E} G} \varphi\left(e^{Y} X\right) e^{-\|Y\|^{2} /(2 h)} d Y .
\end{gathered}
$$

В силу известного свойства интеграла Пуассона в $\mathbb{R}^{m}$

$$
\frac{1}{h}\left(\frac{1}{(\sqrt{2 \pi h})^{m}} \int_{\mathbb{R}^{m}} \psi(Y) e^{-\|Y\|^{2} /(2 h)} d Y-\psi(0)\right)=\frac{1}{2} \Delta \psi(0)+o(1) \quad(h \rightarrow 0) .
$$

Положим $\psi(Y)=\varphi\left(e^{Y} X\right), Y \in T_{E} G$. Тогда

$$
\begin{aligned}
\frac{\left(A_{h} \varphi\right)(X)-\left(A_{0} \varphi\right)(X)}{h} & =\frac{1}{h}\left(\frac{1}{(\sqrt{2 \pi h})^{m}} \int_{\mathbb{R}^{m}} \psi(Y) e^{-\|Y\|^{2} /(2 h)} d Y-\psi(0)\right)+O\left(h^{1 / 2}\right) \\
& =\frac{1}{2} \Delta \psi(0)+o(1) \quad(h \rightarrow 0) .
\end{aligned}
$$

По предложениям 2 и 1

$$
\Delta \psi(0)=\sum_{i=1}^{m} \frac{\partial^{2} \psi}{\left(\partial Y_{i}\right)^{2}}=\sum_{i=1}^{m} \frac{d^{2}}{d t^{2}} \psi\left(Y_{i} t\right)=\sum_{i=1}^{m} \frac{d^{2}}{d t^{2}} \varphi\left(e^{Y_{i} t} X\right)=\Delta \varphi(X),
$$

где $Y_{i}$ - ортонормированный базис в $T_{E} G$. По $(3.18)$ и $(3.19)(P \varphi)(X)=\frac{1}{2}(\Delta \varphi)(X)$.

Для доказательства первого утверждения теоремы достаточно заметить, что на функциях $\varphi \in D_{P}$ полугруппа $A_{t}$ сильно непрерывна, а нами уже доказано, что $D_{P}$ cодержит $C^{2}(G)$ - плотное подпространство в $C(G)$. Известно (см., например, [14, гл. 10]), что подпространство, на котором сжимаюшая полугруппа сильно непрерывна, замкнуто; следовательно, в нашем случае оно совпадает с $C(G)$. Теорема доказана.

Чтобы доказать формулу для решения уравнения теплопроводности, нам осталось проверить, что $A_{t} \varphi \in C^{2}(G)$ для всех $\varphi \in C^{2}(G)$ и $t>0$. 
Лемма 14. 1. Пусть $\varphi \in C^{n}(G)$. Тогда $A_{t} \varphi \in C^{n}(G)$. 2. Eсли $W(X)-$ левоинвариантное векторное поле на группе $G$, т.е. $W(X)=X W(E), W(E) \in T_{E} G$, mо $\nabla_{W}\left(A_{t} \varphi\right)=A_{t}\left(\nabla_{W} \varphi\right)$ для любой функиии $\varphi \in C^{1}(G)$.

ДокАЗАТЕЛьСтво. Сначала мы докажем, что для любого разбиения $T$ отрезка $[0, t]$ дифференцирование вдоль левоинвариантного векторного поля $W(X)$ и оператор $A_{t}^{T}$ коммутируют, т.е. $\nabla_{W}\left(A_{t}^{T} \varphi\right)=A_{t}^{T}\left(\nabla_{W} \varphi\right)$ для любой функции $\varphi \in C^{1}(G)$.

Заметим, что

$$
\int_{G} \nabla_{W} f(X) d X=\lim _{t \rightarrow 0} \int_{G} \frac{1}{t}\left(f\left(X e^{W t}\right)-f(X)\right) d X=0
$$

для левоинвариантного векторного поля $W$; следовательно, справедлива формула интегрирования по частям:

$$
\int_{G} g(X) \nabla_{W} f(X) d X=-\int_{G} f(X) \nabla_{W} g(X) d X .
$$

Теперь заметим, что производные по $X$ и по $Y$ вдоль $W$ от функции $p(X, Y, h)$ связаны соотношением

$$
\begin{aligned}
\nabla_{W(X)} p(X, Y, h) & =\left.\frac{d}{d t}\right|_{t=0} p\left(X e^{W t}, Y, h\right)=\left.\frac{d}{d t}\right|_{t=0} p\left(X, Y e^{-W t}, h\right) \\
& =-\nabla_{W(Y)} p(X, Y, h) .
\end{aligned}
$$

Пользуясь (3.20) и (3.21), получаем следующее:

$$
\begin{aligned}
& \nabla_{W(X)}\left(A_{t}^{T} \varphi\right)(X)=\nabla_{W(X)} \int_{G} \cdots \int_{G} \varphi\left(X_{1}\right) p\left(X_{1}, X_{2}, h_{1}\right) \cdots p\left(X_{k}, X, h_{k}\right) d X_{1} \cdots d X_{k} \\
& \quad=-\int_{G} \cdots \int_{G} \varphi\left(X_{1}\right) p\left(X_{1}, X_{2}, h_{1}\right) \cdots \nabla_{W\left(X_{k}\right)} p\left(X_{k}, X, h_{k}\right) d X_{1} \cdots d X_{k}=\cdots \\
& \quad=-\int_{G} \cdots \int_{G} \varphi\left(X_{1}\right) \nabla_{W\left(X_{1}\right)} p\left(X_{1}, X_{2}, h_{1}\right) \cdots p\left(X_{k}, X, h_{k}\right) d X_{1} \cdots d X_{k} \\
& \quad=\int_{G} \cdots \int_{G} \nabla_{W\left(X_{1}\right)}\left(\varphi\left(X_{1}\right)\right) p\left(X_{1}, X_{2}, h_{1}\right) \cdots p\left(X_{k}, X, h_{k}\right) d X_{1} \cdots d X_{k} \\
& =A_{t}^{T}\left(\nabla_{W} \varphi\right)(X), \quad \varphi \in C^{1}(G) .
\end{aligned}
$$

Далее возьмем последовательность разбиений $T_{n}$ отрезка $[0, t]$ таких, что $\Delta T_{n} \rightarrow 0$. Тогда $\left(A_{t}^{T_{n}} \varphi\right)(X) \underset{X}{\rightrightarrows}\left(A_{t} \varphi\right)(X)(n \rightarrow \infty)$ и

$$
\left(\nabla_{W}\left(A_{t}^{T_{n}} \varphi\right)\right)(X)=\left(A_{t}^{T_{n}}\left(\nabla_{W} \varphi\right)\right)(X) \underset{X}{\rightrightarrows}\left(A_{t}\left(\nabla_{W} \varphi\right)\right)(X) \quad(n \rightarrow \infty)
$$

следовательно, функция $A_{t} \varphi$ дифференцируема вдоль векторного поля $W$ и $\nabla_{W}\left(A_{t} \varphi\right)=$ $A_{t}\left(\nabla_{W} \varphi\right)$. Таким образом, мы доказали второе утверждение леммы.

Фиксируем теперь ортонормированнный базис $E_{1}, \ldots, E_{m}$ в пространстве $T_{E} G$. Левоинвариантные векторные поля $W_{i}(X)=X E_{i}$ образуют ортонормированньй базис в 
каждой точке $X \in G$. Произвольное гладкое векторное поле $V(X), X \in G$, можно разложить по базису: $V(X)=a_{1}(X) W_{1}(X)+\cdots+a_{m}(X) W_{m}(X)$, где $a_{i}(X)=\left(V(X), W_{i}(X)\right)$ - гладкие функции. В таком случае

$$
\begin{aligned}
\left(\nabla_{V}\left(A_{t}^{T_{n}} \varphi\right)\right)(X)= & \sum_{i=1}^{m} a_{i}(X)\left(\nabla_{W_{i}}\left(A_{t}^{T_{n}} \varphi\right)\right)(X)=\sum_{i=1}^{m} a_{i}(X)\left(A_{t}^{T_{n}}\left(\nabla_{W_{i}} \varphi\right)\right)(X) \\
& \underset{X}{\rightrightarrows} \sum_{i=1}^{m} a_{i}(X)\left(A_{t}\left(\nabla_{W_{i}} \varphi\right)\right)(X) \quad(n \rightarrow \infty) ;
\end{aligned}
$$

следовательно, функция $A_{t} \varphi$ дифференцируема вдоль векторного поля $V$ и

$$
\nabla_{V}\left(A_{t} \varphi\right)=\sum_{i=1}^{m} a_{i} A_{t}\left(\nabla_{W_{i}} \varphi\right)
$$

Значит, $A_{t} C^{n}(G) \subseteq C^{n}(G)$. Лемма доказана.

Если $\varphi(X) \in C^{2}(G)$, то $\left(A_{t} \varphi\right)(X)$ дважды дифференцируема по $X$ для всякого $t$. Из теоремы 4 вытекает, что для любого $t \geqslant 0\left(A_{t} \varphi\right)(X)$ дифференцируема по $t$ и

$$
\frac{\partial A_{t} \varphi}{\partial t}=P\left(A_{t} \varphi\right)=\frac{1}{2} \Delta \varphi
$$

Доказательство основного результата этой работы завершено.

\section{СПИСОК ЦИТИРОВАННОЙ ЛИТЕРАТУРЫ}

[1] Йосида К. Функциональный анализ. М.: Мир, 1967.

[2] Хейер Х. Вероятностные меры на локально компактных группах. М.: Мир, 1981.

[3] Malliavin M., Malliavin P. Integration on loop groups. Quasi-invariant measures // JFA. 1990. V. 93. P. 207-237.

[4] Malliavin M., Malliavin P. Integration on loop groups. Asymptotic Peter-Weyl orthogonality // JFA. 1992. V. 108. P. 13-46.

[5] Leandre R. Integration by parts formulas and rotationally invariant Sobolev calculus on free loop spaces // J. Geom. Phys. 1993. №11. P. 517-528.

[6] Airault H., Malliavin P. Integration on loop groups. Heat equation for the Wiener measure // JFA. 1992. V. 104. P. 71-109.

[7] Albeverio S., Hoegh-Krohn R. The energy representation of a Sobolev Lie Group // Compositive Math. 1978. V. 36. P. 37-52.

[8] Барут А., Рончка Р. Теория представлений групп и ее приложения. М.: Мир, 1980.

[9] Feynmann R. Space-time approach to non-relativistic quantum mechanics // Rev. Mod. Phys. 1948. V. 20. P. 367.

[10] Nelson E. Feynmann integrals and the Schrödinger equations // J. Math. Phys. 1964. V. 5. P. 332 .

[11] Смолянов О.Г. Гладкие меры на группах петель // Докл. РАН. 1995. Т. 345. № 4. C. $455-458$.

[12] Уорнер Ф. Основы теории гладких многообразий и групп Ли. М.: Бибфизмат, 1990.

[13] Винберг Э. Б. Компактные группы Ли. М.: Изд-во МГУ, 1967.

[14] Вентцель А. Д. Курс теории случайных процессов. М.: Наука, 1996. 